\title{
The role of surgery in hereditary breast cancer prevention
}

Breast cancer prevention methods in high-risk women include strict screening, preventive chemotherapy and surgery.

The usual surveillance for high-risk women consists in a physical examination, a yearly mammogram starting at 25 -the effectiveness of magnetic resonance in younger women is being speculated with- and transvaginal echography to discard the possibility of ovarian cancer. The high density in these women's breasts and the difficulty in diagnosing ovarian cancer early make it usual to find tumors in the ovaries and breast in an undesirable stage. Up to now, periodic determination of tumoral markers has not been considered.

Preventive chemotherapy in breast cancer is a proven fact. Treatment with tamoxifen on surgically treated patients during 5 years reduces contralateral breast cancer risk by $40 \%{ }^{1}$. The benefits of using it in highrisk patients that have not developed cancer has already been proven in the USA, where it has been administered to these patients since $1998^{2}$. In the European Community trials undertaken had not had these results, but for the first time in 2002 a British publication reports they obtained the same results as the Ameri$\operatorname{can}^{3}$.

Traditionally, women eligible for preventive breast cancer surgery are those with benign lesions considered high-risk, which include:

1) Lobular neoplasia (lobular carcinoma in situ).

2) Ductal hyperplasia or atypical lobular hyperplasia.

3) Multiple papillomatosis.

4) Some types of proliferative fibrocystic mastopathy, difficult to follow-up, especially, in cases with a family history of breast cancer and cancerophobia.

5) Primary breast phyllode tumor and relapses.

For years it has been known that BRCA1 and BRCA2 mutations significantly increase the risk of developing breast cancer. BRCA1 is located on chromosome 17 and is associated with $45 \%$ of hereditary breast cancer and with $85 \%$ of breast and ovarian cancer syndromes. Its mutation affects $0.0006 \%$ of the population (one in every 1,600) and is the cause of breast cancer in $5 \%$ of women under 40 , in $2 \%$ of women between 40 and 49, and in 1\% of those aged between 50 and 70 . There is an $85 \%$ risk of developing contralateral breast cancer in BRAC1 mutation carriers among women aged $70^{4}$. Specific mutations of this ge- ne have been found in certain groups, among them Ashkenazi and Sephardic Jews and some Eskimo tribes. It must be pointed out that the behaviour of breast cancer in women with this type of gene mutation is more favorable than other type of hereditary cancer. BRCA2, less studied so far, is located on chromosome 17 and causes $40 \%$ of hereditary breast cancer. Other genes implied in hereditary breast cancer are p53 and the ataxia-telangiectasia gene.

There is no agreement on the type of surgery that should be used to treat high-risk women. It seems paradoxical, when a universal consensus has been reached about the validity of conservative breast surgery in invasive cancer, that bilateral mastectomy is advocated when a cancer has not appeared and may never appear. Less aggressive interventions, like skin-sparing mastectomy or subcutaneous mastectomy, that preserve all the skin and do not remove all breast tissue, are not criticized for being less safe.

Bilateral prophylactic mastectomy is being tested on $B R C A 1$ and $B R C A 2$ mutation carriers to prove that it does reduce the risk of breast cancer. A recent analysis done at Rotterdam University on a group of 139 patients, out of which 76 underwent bilateral mastectomy and 63 were placed under surveillance, concludes that after 3 years this intervention reduces the incidence of breast cancer 5 .

Breast reconstruction after a mastectomy is not a minor problem. To recuperate breast volume the patient's own tissues or an implant can be used. The advantage of using the patient's own tissues is better tolerance; the inconveniences, a more complex surgical intervention, more scars and worse aesthetic results. Implants are easier to use, but are sometimes not well tolerated -periprosthetic capsules may be formed- they can be rejected and are usually prone to a greater number of surgical infections. The areola-nipple complex also poses problems for reconstruction surgery, which are solved by means of complicated technical devices. The final result is often unsatisfactory for both the patient and the surgeon. There is no agreement either on the timing of reconstruction surgery. Some advocate performing it just after mastectomy, others prefer a differed intervention.

Skin-sparing mastectomy is a less radical mastectomy with regards to its capacity to eliminate the totality of glandular tissue ${ }^{6}$. The skin removed is limited to the 
areola-nipple complex and to a variable in size spindle of skin, which includes the projection surface of the tumor. This technique allows creating mammary volume with the patient's own skin to cover the implant. It has a more aesthetic result.

Subcutaneous or subdermic mastectomy or adenomastectomy, consists in the exeresis of the glandular tissue totally or partially, preserving the breast cutaneous cover, including the areola and the nipple, eliminating the skin or not, depending on aesthetic reasons. Liberating the mammary parenchyma from the thin adipose layer which separates it from the skin, makes it difficult to remove it completely keeping the skin viable. On the other hand, the nipple has to be considered mammary tissue and to avoid necrosis, a small amount of gland has to be left under it. Therefore, even when done with the best oncological intention, it must be accepted that subcutaneous mastectomy leaves $10 \%$ of the mammary parenchyma, which is susceptible of malignancy. Breast volume is usually obtained by using implants. Recently endoscope-assisted procedures have been introduced with apparently better aesthetic results ${ }^{7}$.

Subcutaneous mastectomy as breast cancer prevention in high-risk patients has been widely studied. Pennisi and Capozzi ${ }^{8}$, analysed 1,500 patients in 1989 and showed that after 10 years, only $6(0,4 \%)$ out of 1,007 patients screened (454 were lost for screening, less than $-30 \%$ loss), developed breast cancer. In a more recent retrospective study conducted by the Mayo Clinic $^{9}$, to prove the results of prophylactic mastectomy in women with a family history of breast cancer, 7 out of 573 patients who underwent bilateral subcutaneous mastectomies presented breast cancer after 14 years screening ( $0.81 \%$ frequency).

This operation, followed by axillary lymphadenectomy has recently been compared with radical mastectomy for extensive intraductal breast cancer, which does not allow conservative procedures. A comparative study of 133 subcutaneous mastectomies and axillary lymphadenectomy with 910 radical mastectomies was undertaken at Gunma University, Japan in 2001. Local relapses were more frequent in the group treated with subcutaneous mastectomy (much younger patients), but survival rates were higher among these than among the mastectomized ones ${ }^{10}$.
Because surgery is not a safe prevention method; because it is a complex procedure and often does not give satisfactory results; because there is no agreement on which procedure is more satisfactory and because it has been recently proven that tamoxifen prevention is more effective in $B R A C 1 B R A C 2$ mutation carriers, where risk reduction is close to $84 \%$ if associated with oophorectomy ${ }^{11}$, it will be difficult to accept surgery as a preventive method for hereditary breast cancer, in spite of being a logical procedure.

\section{Bibliografía}

1. O'Regan RM, Jordan VC, Gradishar WJ. Tamoxifen and contralateral breast cancer. J Am Coll Surg 1999; 188:678-83.

2. Fisher B, Constantino JP, Wickerham DL, et al. Tamoxifen for prevention of breast cancer: Report of the National Surgical Adjuvant Breast and Bowel Project P-1 study. J Natl Cancer Inst 1998; 90: 1371-88.

3. IBIS investigators. First results from the International Breast Cancer Intervention Study (IBIS-I): a randomized prevention trial. Lancet 2002; 360: 817-24.

4. Ford D, Easton DF, Bishop DT, Narod SA, Godgar DA. Risks of cancer in BRCA1 mutation carriers. Lancet 1994; 342: 692-5.

5. Meijers-Heijboer H, van Geel B, van Putten W, et al. Breast cancer after prophylactic bilateral mastectomy in women with a BRCA1 or BRCA2 mutation. N Engl J Med 2001; 345: 159-64.

6. Kroll SS, Khoo A, Singletary SE, et al. Local recurrence risk after skin-sparing and conventional mastectomy: a 6-year follow-up. Plast Reconstr Surg 1999; 104: 421-5.

7. Kitamura K, Ishida M, Inoue H, Hinoshita J, Hashizume M, Sugimachi K. Early results of an endoscope-assisted subcutaneous mastectomy and reconstruction for breast cancer. Surgery 2002; 131: S324-9.

8. Pennisi VR, Capozzi A. Subcutaneous Mastectomy Data: A Final Statistical Analysis of 1500 Patients. Aesth Plast Surg 1989; 13: 15-21.

9. Hartmann LC, Schaid DJ, Woods JE, et al. Efficacy of bilateral prophylactic mastectomy in women with a family history of breast cancer. N Engl J Med 1999; 340: 77-84.

10. Horiguchi J, Iino Y, Takei H, et al. A Comparative Study of Subcutaneous Mastectomy with Radical Mastectomy. Anticancer Res 2001; 21: 2963-8.

11. Narod SA, Brunet JS, Ghadirian P, et al. Tamoxifen and risk of contralateral breast cancer in BRCA1 y BRCA2 mutation carriers: a case-control study. Lancet 2000; 356: 1876-81.

José Díaz-Faes Fundación de Estudios Mastológicos 